EESTI NSV TEADUSTE AKADEEMIA TOIMETISED. XI KÖDE

FOOSIKALIS-MATEMAATILISTE JA TEHNILISTE TEADUSTE SEERIA. 1962, NR. 4

ИЗВЕСТИЯ АКАДЕМИИ НАУК ЭСТОНСКОЙ ССР. ТОМ ХІ СЕРИЯ ФИЗИКО-МАТЕМАТИЧЕСКИХ И ТЕХНИЧЕСКИХ НАУК. 1962, № 4

\title{
О ДВУХ ТЕОРЕМАХ ЧЖОУ И ИХ ОБОБЩЕНИЯХ НА ДВОЙНЫЕ РЯДЫ
}

\section{С. БАРОН,}

\section{кандидат физико-математических наук}

\section{Э. ПАЛЛУМ}

\section{M. ПЕТЕРСOH}

\section{$\S 1$. Введение}

В практике часто приходится иметь дело с рядами, которые не суммируемы методами Чезаро. Оказывается, что и для таких рядов можно найти некоторые типы множителей суммируемости. Для этой цели вводим вспомогательную последовательность положительных чисел при помощи следующих определений.

Ряд *

$$
\Sigma u_{n}
$$

называем $\left|C_{\varphi}^{\alpha}\right|$-суммируемым, если ряд $\Sigma \varphi_{n} u_{n}^{\prime}$, где

$$
u_{n}^{\prime}=\sum_{k=0}^{n} \frac{k A_{n-k}^{\alpha-1}}{n A_{n}^{\alpha}} u_{k},
$$

абсолютно сходится.

Последовательность $\left\{U_{n}\right\}$ называем $C_{\varphi}^{\alpha}$-ограниченной ( $C_{\varphi}^{\alpha}$-суммируемой), если последовательность $\left\{\varphi_{n} U_{n}^{\prime}\right\}$, где

$$
U_{n}^{\prime}=\sum_{k=0}^{n} \frac{A_{n-k}^{\alpha-1}}{A_{n}^{\alpha}} U_{k}
$$

ограничена (сходится).

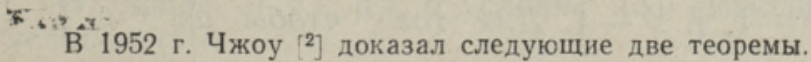

Те орем а I. Пусть $\left\{\varphi_{n}\right\}$ такая последовательность положительных чисел, что $n^{-1} \varphi_{n}$ не возрастает $и \alpha, \beta \geqslant 0$. Для того, чтобы из $\left|C_{\varphi}^{\alpha}\right|$-суммируемости ряда (1) всегда следовала $\left|C^{\beta}\right|$-суммируемость ряда

* Если не оговорено противное, то в $\$ 1-3$ сохраняем определения и обозначения статьи [4], а в $\$ 4$ и 5 - определения и обозначения статей [ $\left.{ }^{5}, 6\right]$. 


$$
\sum \varepsilon_{n} \bar{u}_{n}
$$

необходимы и достаточны условия

$$
\begin{aligned}
\varepsilon_{n} & =O\left(n^{\beta-\alpha} \varphi_{n}\right), \\
\varepsilon_{n} & =O\left(\varphi_{n}\right) \\
\Delta^{\alpha}\left(n^{-1} \varepsilon_{n}\right) & =O\left(n^{-\alpha-1} \varphi_{n}\right) .
\end{aligned}
$$

Теорем а II. Пусть $\varphi_{n}=(n+1)-p, \alpha, \beta, p \geqslant 0$. Для того, чтобы из $C_{\varphi}^{\alpha}$-ограниченности последовательности

$$
\left\{n u_{n}\right\}
$$

всегда следовала $\mid C^{\beta}$ |-суммируемость ряда (2), необходимы и достаточны условия

$$
\begin{aligned}
& \Sigma(n+1)^{\alpha-1-\beta+p}\left|\varepsilon_{n}\right|<\infty, \\
& \Sigma(n+1)^{-1}+p\left|\varepsilon_{n}\right|<\infty, \\
& \sum_{n=1}^{\infty} n^{\alpha+p}\left|\Delta^{\alpha}\left(n^{-1} \varepsilon_{n}\right)\right|<\infty .
\end{aligned}
$$

В 1956 г. Бозанкет и Чжоу (см. ['], стр. 77) показали, что в теореме I, если $\varphi_{n}$ не возрастает, условие (J') можно заменить условием

$$
\Delta^{\alpha} \varepsilon_{n}=O\left(n^{-\alpha} \varphi_{n}\right)
$$

а в теореме II условие (B') условием

$$
\Sigma(n+1)^{\alpha-1+p}\left|\Delta^{\alpha} \varepsilon_{n}\right|<\infty .
$$

В настоящей статье мы дадим новые, более простые док̈азательства теорем I и II в формулировке статьи [1] (причем в более общем виде), а затем обобщим их на двойные ряды. Для доказательства применим метод статьи [4].

Итак, целью настоящей статьи является доказательство следующих, теорем и их обобщение на двойные ряды.

Те орем а 1. Пусть * $\left\{\varphi_{n}\right\}-$ невозрастающая последовательность

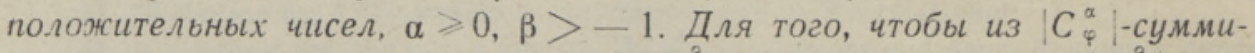
руемости ряда (1) всегда следовала $C^{3}$-суммируемость (или $\left|C^{\beta}\right|$-суммируемость) ряда (2), необходимы и достаточны условия (D), (D') $и$ (J).

Т еорем а 2. Пусть $\left\{\varphi_{n}\right\}$ - невозрастающая последовательность положительных чисел, $\alpha \geqslant-1, \beta>-1$. Для того, чтобы из ** $C_{\varphi}^{\alpha+1}-$

* При $\varphi_{n}=1$ теорема 1 переходит в теорему 1 статьи [4] (если $\left.\beta \geqslant 0\right)$ и теорему 1 статьи [7] (если $-1<\beta<0)$.

[12-15], в которых показывается, что условия теоремы 2 необходимы и достаточны для того, чтобы из $C_{\varphi}^{\alpha}$-ограниченности частичных сумм ряда (1) всегда следовала $\left|C^{\beta}\right|$ суммируемость ряда (2). Однако достаточность этих условий, доказательство чего больше всего занимает место в $\left[{ }^{12-15}\right]$, следует из теоремы 2 при помощи формуль (6.1.4) книги $\left[{ }^{10}\right]$ (ср. ['], стр. 82). 
ограниченности (или $C_{\varphi}^{\alpha+1}$-суммируемости) последовательности (3) всегда следовала $\left|C^{3}\right|$-суммируемость ряда (2), необходимы и достаточны условия

$$
\begin{aligned}
& \Sigma(n+1)^{\alpha-\beta} \varphi_{n}^{-1}\left|\varepsilon_{n}\right|<\infty, \\
& \Sigma(n+1)^{-1} \varphi_{n}^{-1}\left|\varepsilon_{n}\right|<\infty, \\
& \Sigma(n+1)^{\alpha} \varphi_{n}^{-1}\left|\Delta^{\alpha+1} \varepsilon_{n}\right|<\infty .
\end{aligned}
$$

3 амеч ание 1. Если-1< $\beta \leqslant 0$, то в теореме 1 можем опустить условия (J) и (D'), а в теореме 2 - условия (В) $и$ (М').

\section{§ 2. Доказательство теоремы 1}

Необходимость. Аналогично, как на стр. 50 статьи $\left[{ }^{4}\right]$, можно доказать:

Для того, чтобы из $\left|C_{\varphi}^{\alpha}\right|$-суммируемости ряда (1) всегда следовала $C^{\beta}$-суммируемость ряда (2), необходимо условие

$$
v A_{v}^{\alpha} \sum_{k=v}^{n} A_{k-v}^{-\alpha-1} \frac{A_{n-k}^{\beta}}{A_{n}^{\beta}} \frac{\varepsilon_{k}}{k}=O\left(\varphi_{v}\right) \quad(n \geqslant v) .
$$

Положив $v=n$ в условии (4), получаем необходимость условия (D).

Положив $\alpha=0$ в условии (4) и переходя в нем к пределу при $n \rightarrow \infty$, в силу включения $\left|C^{0}\right| \subset C^{\alpha} \mid$, сразу получаем необходимость условия (D'), откуда, так как $\varphi_{n}$ не возрастает, в свою очередь, вытекает условие

$$
\varepsilon_{n}=O(1)
$$

Учитывая необходимость условия (A), мы можем в условии (4) перейти к пределу при $n \rightarrow \infty$, вследствие чего получаем необходимость условия (J'). Наконец, из условий (A) и (J') следует условие (J) (см., например, [1], стр. 79).

Д ост аточность. Как показал Чжоу ([2], стр. 465)

Для того, чтобы из $\left|C_{\varphi}^{z}\right|$-суммируености ряда (1) всегда следовала $\mid C^{\xi}$ |-суммируемость ряда (2), необходимо и достаточно условие

$$
\sum_{n=\mathrm{v}}^{\infty} \frac{1}{n A_{n}^{\beta}}\left|\sum_{k=v}^{n} A_{k-\nu}^{-\alpha-1} A_{n-k}^{\beta-1} \varepsilon_{k}\right|=O\left(v^{-\alpha-1} \varphi_{v}\right) .
$$

В случае $-1<\beta<0$ условие (5) выводится непосредственно из условия (D).

Пусть теперь $0 \leqslant \beta \leqslant \alpha$. Для этого случая остается доказать, что из условий (D), (D') и (J) следует условие (5). Для доказательства последнего можно применять рассуждения, аналогичные приведенным на стр. 50-54 статьи [ $\left.{ }^{4}\right]$, заменяя лемму 2 этой статьи леммой 13 из статьи $\left[{ }^{2}\right]$.

Случай $\beta>\alpha$ очевиден в силу включения $\left|C^{\alpha}\right| \subset\left|C^{\beta}\right|$. 


\section{§ 3. Доказательство теоремы 2}

Аналогично, как на стр. 60 статьи [3], можно доказать:

Для того, чтобы из $C_{\varphi}^{\alpha+1}$-ограниченности (или $C_{\varphi}^{\alpha+1}$-суммируемости) последовательности (3) всегда следовала $\left|C^{\beta}\right|$-суммируемость ряда (2), необходимо и достаточно условие

$$
\sum_{\nu=1}^{\infty} \frac{1}{\varphi_{\nu}} A_{\nu}^{\alpha+1}\left|\sum_{n \in \Re} \frac{1}{n A_{n}^{\beta}} \sum_{k=v}^{n} A_{k-\nu}^{-\alpha-2} A_{n-k}^{\beta-1} \varepsilon_{k}\right| \leqslant K
$$

где $\Re$ - всевозможные конечные подмножества последовательности натуральных чисел, а К не зависит от $\Re$.

Необходй мость. Положив $n=v$ в условии (6), что допустимо по теореме 1 статьи $\left[{ }^{3}\right]$, получаем необходимость условия $(M)$.

Положив $\alpha=-1$ в условии (6), в силу включения $C^{0} \subset C^{\alpha+1}$, получаем необходимость условия

$$
\lim _{p} \sum_{\nu=1}^{l} \frac{1}{\varphi_{\nu}}\left|\varepsilon_{\nu}\right|\left|\sum_{n=v}^{p} \frac{1}{n A_{n}^{\beta}} A_{n-v}^{\beta-1}\right| \leqslant K \quad(l=1,2, \ldots),
$$

равносильное условию (M').

Далее, из условия (6), в силу условия * (M'), выводим

$$
\begin{aligned}
& \lim _{p} \sum_{\nu=1}^{l} \frac{1}{\varphi_{v}} A_{v}^{\alpha+1}\left|\sum_{n=v}^{p} \frac{1}{n A_{n}^{\beta}} \sum_{k=v}^{n} A_{k-\nu}^{-\alpha-2} A_{n-k}^{\beta-1} \varepsilon_{k}\right|= \\
= & \sum_{v=1}^{l} \frac{1}{\varphi_{v}} A_{v}^{\alpha+1}\left|\Delta^{\alpha+1} \frac{\varepsilon_{v}}{v}\right| \leqslant M \quad(l=1,2, \ldots),
\end{aligned}
$$

откуда, ввиду включения $C^{\alpha} \subset C^{\alpha+1}(\alpha>-1)$ и вытекающего из леммы 1 статьи [ $\left.{ }^{1}\right]$ соотношения **

$\sum_{\nu=1}^{\infty} \frac{1}{\varphi_{\nu}} v^{\alpha}\left|\Delta^{\alpha+1} \varepsilon_{\nu}\right|=O(1) \sum_{\nu=1}^{\infty} \frac{1}{\varphi_{\nu}} v^{\alpha+1}\left|\Delta^{\alpha+1} \frac{\varepsilon_{v}}{\nu}\right|+(\alpha+1) \sum_{\nu=1}^{\infty} \frac{1}{\varphi_{\nu}} v^{\alpha}\left|\Delta^{\alpha} \frac{\varepsilon_{\nu}}{\nu}\right| \leqslant M$, заключаем необходимость условия (В).

Достаточность. В случае $-1<\beta<0$ условие (6) выводится непосредственно из условия $(M)$.

Пусть $0 \leqslant \beta \leqslant \alpha+1$. Для этого случая остается доказать, что из условий $(M),\left(M^{\prime}\right)$ и (B) следует условие (6). Доказательство последнего аналогично доказательству достаточности теоремы 4 статьи [4], однако намного проще его, так как в нашем случае вовсе не надо отдельно рассматривать выражения, соответствующие суммам $P, Q, T$ и

* Условие $\left(M^{\prime}\right)$ гарантирует существование предела и законность перестановки шорядка суммирования в (8).

** См. ['], стр. 80. При $-1<\alpha<0$ сходимость крайнего ряда справа обеспечено условием (M') (ср. ['], лемма 5). 
$V$ (см. [4], стр. 63 и 66 ). В нашем доказательстве при $\alpha \geqslant 0$ нам нужны лемма 7 статьи ${ }^{[4]}$ и следующая *

Лем м а 1. Из условий (А) и (В) следует

$$
\Sigma(n+1)^{\sigma-1} \varphi_{n}^{-1}\left|\Delta^{\sigma} \varepsilon_{n}\right| \leqslant \Sigma(n+1)^{\alpha} \varphi_{n}^{-1}\left|\Delta^{\alpha+1} \varepsilon_{n}\right| \quad(1 \leqslant \sigma \leqslant \alpha+1) .
$$

Доказ ательство см. [11], стр. 194-195.

Случай $\beta>\alpha+1$ очевиден в силу включения $\left|C^{\alpha+1}\right| \subset\left|C^{\beta}\right|$.

3 а меч ан ие 2 . Положив в теореме $2 \varphi_{n}=1$ и пользуясь формулой (6.1.6) в книге [10], получаем более простое доказательство достаточности условий теоремы 4 статьи [ $\left.{ }^{4}\right]$. В частности, отсюда получаем обобщения последней теоремы и теоремы 2 статьи [7] и для $\alpha>-1$, так как необходимость их условий при $-1<\alpha<0$ вытекает из условия (24) статьи $\left[{ }^{4}\right]$ (без применения условия (А)).

\section{§4. Обобщение теорем 1 и 2 на двойные ряды}

Двойной ряд

$$
\sum_{m, n}^{\Sigma} u_{m n}
$$

называем $l$ - $C_{\psi}^{\alpha, \beta}$-суммируемым, если двойной ряд $\sum_{m, n} \psi_{m n} u_{m n}^{\prime}$,

где

$$
u_{m n}^{\prime}=\sum_{k, l=0}^{m, n} \frac{k l}{m n} \frac{A_{m-k}^{\alpha-1} A_{n-l}^{\beta-1}}{A_{m}^{\alpha} A_{n}^{\beta}} u_{k l}
$$

абсолютно сходится.

Двойную последовательность $\left\{U_{m n}\right\}$ называем $C_{\psi}^{\alpha, \beta}$-ограниченной $\left(b \cdot C_{i}^{\alpha, \beta}\right.$-суммируемой, $\quad r-C_{\psi}^{\alpha, \beta}$-суммируемой), если последовательность $\left\{\psi_{m n} U_{m n}^{\prime}\right\}$, где

$$
U_{m n}^{\prime}=\sum_{k, l=0}^{m, n} \frac{A_{m-k}^{\alpha-1} A_{n-l}^{\beta-1}}{A_{m}^{\alpha} A_{n}^{\beta}} U_{k l}
$$

ограничена (b-сходится, $r$-сходится).

Соответственно теоремам 1 и 2 в случае двойных рядов имеют место следующие теоремы.

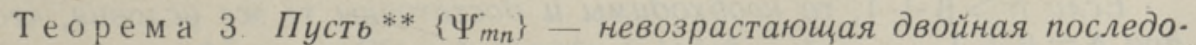
вательность положительных чисел, $\alpha, \beta \geqslant 0, \gamma, \delta>-1$. Если $\gamma, \delta \leqslant$ $\leqslant \alpha, \beta$, то для того, чтобы из $l$ - $C_{i}^{\alpha, \beta}$-суммируемости ряда (9) всегда

* При $-1 \leqslant \boldsymbol{\alpha}<0$ названные леммы не используются, $a=-1, b=0$, а соотношение $\Delta \varepsilon_{n}=\Delta^{-\alpha}\left(\Delta^{x+1} \varepsilon_{n}\right)$ (для преобразования суммы $F^{\prime}$ ) следует из условия (M').

** При $\psi_{m n}=1$ теорема 3 переходит в теорему 3 статьи [5] (если $\left.\gamma, \delta \geqslant 0\right)$ и теорему 3 статьи [7] (если $\gamma<0$ или $\delta<0$ ). 
следовала $C^{\gamma, \hat{\jmath}}$-суммируемость $\left(C_{b}^{\gamma, \hat{\jmath}}\right.$-суммируемость, $C_{r}^{\gamma, \hat{\jmath}}$-суммируемость или $C_{l}^{\gamma, \hat{\jmath}}$-суммируемость) ряда

$$
\sum_{m, n} \varepsilon_{m n} u_{m n}
$$

необходимы и достаточны условия

$$
\begin{aligned}
\varepsilon_{m n} & =O\left[(m+1)^{\gamma-\alpha}(n+1)^{\delta-\beta} \psi_{m n}\right], \\
\Delta_{m}^{\alpha} \varepsilon_{m n} & =O\left[(m+1)^{-\alpha}(n-1)^{\delta-\beta} \psi_{m n}\right], \\
\Delta_{n}^{\beta} \varepsilon_{m n} & =O\left[(m+1)^{\gamma-\alpha}(n+1)^{-\beta} \psi_{m n}\right], \\
\Delta_{m n}^{\alpha \beta} \varepsilon_{m n} & =O\left[(m+1)^{-\alpha}(n+1)^{-\beta} \psi_{m n}\right] .
\end{aligned}
$$
$\delta=\beta$.

Если $\delta>\beta$, то необходимы и достаточны те же условия, что и при

Если $\gamma>\alpha$, то необходимы и достаточны те же условия, что и при $\gamma=\alpha$.

Т еорем а 4. Пусть $\left\{\psi_{m n}\right\}$ - невозрастающая двойная последовательность положительных чисел, $\alpha, \beta \geqslant-1, \gamma, \delta>-1$. Если $\gamma, \delta \leqslant$ $\leqslant \alpha+1, \beta+1$, то для того, чтобы из $C_{\psi}^{a+1, \beta+1}$-ог̆раниченности (или $b-C_{\psi}^{\alpha+1, \beta+1}$-суммируемости, или $r-C_{\psi}^{\alpha+1, \beta+1}-$ суммируемости) последовательности

$$
\left\{m n u_{m n}\right\}
$$

всегда следовала $C_{l}^{\gamma, \delta}$-суммируемость ряда (10), необходимы и достаточны условия

$$
\begin{aligned}
& \sum_{m, n}(m+1)^{\alpha-\gamma}(n+1)^{\beta-\delta} \psi_{m n}^{-1}\left|\varepsilon_{m n}\right|<\infty, \\
& \sum_{m, n}(m+1)^{\alpha}(n+1)^{\beta-\delta} \psi_{m n}^{-1}\left|\Delta_{m}^{\alpha+1} \varepsilon_{m n}\right|<\infty, \\
& \sum_{m, n}(m+1)^{\alpha-\gamma}(n+1)^{\beta} \psi_{m n}^{-1}\left|\Delta_{n}^{\beta+1} \varepsilon_{m n}\right|<\infty, \\
& \sum_{m, n}(m+1)^{\alpha} .(n+1)^{\beta} \psi_{m n}^{-1}\left|\Delta_{m n}^{\alpha+1 \beta+1} \varepsilon_{m n}\right|<\infty .
\end{aligned}
$$

Если $\delta>\beta+1$, то необходимы и достаточны те же условия, что и при $\delta=\underline{\beta}+1$.

Если $\gamma>\alpha+1$, то необходимь и достаточны те же условия, что и при $\gamma=\alpha+1$.

3 а меч ан ие 3. Если -1 $<\gamma \leqslant 0$, то в теореме 3 можем опустить условия $\left(\mathrm{K}_{1}\right)$ и (J), а в теореме 4 - условия $\left(\mathrm{L}_{1}\right)$ и (В). Если $-1<\delta \leqslant 0$, то в теореме 3 можем опустить условия $\left(\mathrm{K}_{2}\right)$ и $(\mathrm{J})$, а в теореме 4 - условия $\left(\mathrm{L}_{2}\right)$ и (B). 


\section{§ 5. Доказательство теоремы 3}

Не обходимость. Аналогично, как теорему 1 статьи [5], можно доказать:

Для того, чтобы из l-C ${ }_{\psi}$, -суммируемости ряда (9) всегда следовала $C^{\gamma, \grave{-}-с у м м и р у е м о с т ь ~ р я д а ~(10), ~ н е о б х о д и м о ~ у с л о в и е ~}$

$$
\mu \nu A_{\mu}^{\alpha} A_{\nu}^{\beta} \sum_{k, l=\mu, \nu}^{m, n} A_{k-\mu}^{-\alpha-1} A_{l-\nu}^{-\beta-1} \frac{A_{m-k}^{\gamma} A_{n-l}^{\gamma}}{A_{m}^{\gamma} A_{n}^{\delta}} \frac{\varepsilon_{k l}}{k l}=O\left(\psi_{\mu \nu}\right) .
$$

При помощи рассуждений, приведенных в 22 данной статьи и в $\S 2$ статьи [5], можем из условия (12) вывести необходимость условий теоремы 3 .

Д ост а точность. Аналогично, как теорему 2 статьи [ $\left.{ }^{5}\right]$, можно доказать:

Для того, чтобы из l-C $C_{\psi}^{\alpha}$-суммируемости ряда (9) всегда следовала $C_{l}^{\gamma, \delta}$-суммируемость ряда (10), необходимо и достаточно условие

$$
\begin{gathered}
\sum_{m, n=\mu, v}^{\infty} \frac{\mu \nu}{m n A_{m}^{\gamma} A_{n}^{\hat{\jmath}}}\left|\sum_{k, l=\mu, \nu}^{m, n} A_{k-\mu .}^{-\alpha-1} A_{l-\nu}^{-\hat{\beta}-1} A_{m-k}^{\gamma-1} A_{n-l}^{\hat{o}-1} \varepsilon_{k l}\right|= \\
=O\left[(\mu+1)^{-\alpha}(\nu+1)^{-\beta} \psi_{\mu \nu}\right] .
\end{gathered}
$$

Остается доказать, что из условий теоремы 3 следует условие (13). Последнее доказывается при помощи рассуждений, приведенных в $\$ 4$ статьи [5] и при доказательстве теоремы 3 статьи [7], заменяя леммы 5 и 6 статьи [5] следующими:

Л ем м 2. Из условий

$$
\varepsilon_{m n}=O(1)
$$

и (J) для всех $0<\sigma, \tau \leqslant \alpha, \beta$ следует

$$
\Delta_{m n}^{\sigma \tau} \varepsilon_{m n}=O\left\{(m+1)^{-\sigma}(n+1)^{-\tau} \psi_{m n}\right] .
$$

Л ем м а З. Из условий (А) $и\left(\mathrm{~K}_{1}\right)$ для всех $0 \leqslant 0 \leqslant$ в вытекает

$$
\Delta_{m}^{\sigma} \varepsilon_{m n}=O\left[(m+1)^{-\sigma}(n+1)^{\delta-\beta} \psi_{m n}\right] .
$$

а из условий (А) и $\left(\mathrm{K}_{2}\right)$ для всех $0<\tau \leqslant \beta$

$$
\Delta_{n}^{\tau} \varepsilon_{m n}=O\left[(m+1)^{\gamma-\alpha}(n+1)^{-\tau} \psi_{m n}\right] .
$$

Доказательства такие же, как у лемм 5 и 6 статьи [5]. 


\section{§6. Доказательство теоремы 4}

Аналогично, как теорему 1 статьи [ $\left.{ }^{6}\right]$, можно доказать:

Для того, чтобы из $C_{\psi}^{\alpha+1, \beta+1}$-ограниченности (или $b-C_{\psi}^{\alpha+1, \beta+1}$-суммируемости, и.ли $r$ - $C_{\psi}^{\alpha+1, \beta+1}$-суммируемости) последовательности (11) всегда следовала С., ${ }^{7,}$-суммируемость ряда (10), необходимо и достаточно условие

$$
\sum_{\mu, \nu} \frac{\mu \nu}{(\mu+1)(v+1)} \frac{1}{\psi_{\mu \nu}} A_{\mu,}^{\alpha+1} A_{\nu}^{\beta+1}\left|\sum_{m, n \in \mathfrak{M i}} \frac{1}{m n A_{m}^{\gamma} A_{n}^{\gamma}} \sum_{k, l=\mu, \nu}^{m, n} A_{k-\mu}^{-\alpha-2} A_{l-\nu}^{-\beta-2} A_{m-k}^{\gamma-1} A_{n-l}^{\delta-1} \varepsilon_{k l}\right| \leqslant K
$$

для любого $\mathfrak{\Re}$, где $\mathfrak{M}$ - конечное множество пар неотрицательных целых чисел, а $K$ не зависит от $\mathfrak{M}$.

Необходимость. Положив $m, n=\mu, v$ в условии (14) (что допустимо, ибо часть I теоремы 1 статьи [3], как видно из ее доказательства, распространяется на двойные ряды), получаем необходимость условия (M).

Положив $\alpha=-1$ и $n=v$ в условии (14), в силу включения * $C_{r}^{0, \beta} \subset C_{r}^{\alpha+1, \beta+1}$, получаем условие, напоминающее (7), откуда следует необходи'мость условия

$$
\sum_{m, n}(m+1)^{-1}(n+1)^{\beta-\delta} \psi_{m n}^{-1}\left|\varepsilon_{m n}\right|<\infty,
$$

а в силу включения $C_{r}^{0.0} \subset C_{r}^{\alpha+1, \beta+1}$, ноложив $\alpha=\beta=-1$ в условии (14), получаем необходимость условия

$$
\sum_{m, n}(m+1)^{-1}(n+1)^{-1} \psi_{m n}^{-1}\left|\varepsilon_{m n}\right|<\infty .
$$

В силу последнего условия, из (14) (положив в нем $n=v$ ) выводим условие, напоминающее (8), откуда, ввиду включения $C_{r}^{\alpha, \beta+1} \subset C_{r}^{\alpha+1, \beta+1}$ $(\alpha>-1)$, заключаем необходимость условия $\left(\mathrm{L}_{1}\right)$, а положив $\beta=-1$ в (14), учитывая (7) и (8), такнм же образом заключим необходимость условия

$$
\sum_{m, n}(m+1)^{\alpha}(n+1)^{-1} \psi_{m n}^{-1}\left|\Lambda_{m}^{\alpha+1} \varepsilon_{m n}\right|<\infty .
$$

Наконец, из условия (14), в силу (15), аналогично (8) выводим

$$
\sum_{\mu, \nu=0}^{k, l} \frac{\mu \nu}{(\mu+1)(\nu+1)} \frac{1}{\psi_{\mu \nu}} A_{\mu}^{\alpha+1} A_{v}^{\beta+1}\left|\Delta_{\mu \nu}^{\alpha+1 \beta+1} \frac{\varepsilon_{\mu \nu}}{\mu \nu}\right| \leqslant K(k, l=0,1, \ldots),
$$

откуда ввиду соответствующих включений и легко доказываемого обобщения на двойные ряды лемм 1 и 5 статьи ['] заключаем необходимость условия (В).

* См. [ $\left.{ }^{8}\right]$, стр. 32 , теорема 6 . 
Необходимость остальных условий теоремы 4 доказывается аналогично.

Дост аточность. Остается доказать, что из условий теоремы 4 следует условие (14). Последнее доказывается при помощи рассуждений, приведенных в $\S 5$ статьи [] (с соответствующи'ми упрощениями, указанными в $\$ 3$ нашей статьи) и при доказательстве теоремы 4 статьи [7], причем в этом доказательстве нам нужны лемма 7 статьи [4], лемма 6 статьи [6] и следующие леммы.

Л емм а 4. Из условий (А) $и$ (В) для всех $0<\sigma, \tau \leqslant \alpha+1, \beta+1$ вытекает

$$
\sum_{m, n}(m+1)^{\sigma-1}(n+1)^{\tau-1} \psi_{m n}^{-1}\left|\Lambda_{m n}^{\sigma-} \varepsilon_{m n}\right|<\infty .
$$

Л ем м а 5 . Из условий (A) $и\left(\mathrm{~L}_{1}\right)$ для всех $0<\sigma \leqslant \alpha+1$ вытекает

$$
\sum_{m, n}(m+1)^{\sigma-1}(n+1)^{\beta-\hat{\sigma}} \psi_{m n}^{-1}\left|\Delta_{m}^{\sigma} \varepsilon_{m n}\right|<\infty,
$$

а из условия (A) и $\left(\mathrm{L}_{2}\right)$ для всех $0<\tau \leqslant \beta+1$

$$
\sum_{m, n}(m+1)^{\alpha-\gamma}(n+1)^{\tau-1} \psi_{m n}^{-1}\left|\Delta_{n}^{\tau} \varepsilon_{m n}\right|<\infty
$$

доказ ательство леммы 4 аналогично доказательству леммы 13 статьи [ $\left.{ }^{9}\right]$ и основывается на лемме 1. Лемма 5 непосредственно вытекает из леммы 1 .

3 амеч ани е 4. Положи'в в теореме $4 \psi_{m n}=1$ и пользуясь формулой

$$
\tau_{m n}^{\alpha+1, \beta+1}=(\alpha+1)(\beta+1)\left(\sigma_{m n}^{\alpha, \beta}-\sigma_{m n}^{\alpha, \beta+1}-\sigma_{m n}^{\alpha+1, \beta}+\sigma_{m n}^{\alpha+1, \beta+1}\right),
$$

вытекающей из формул (3.9), (2.3), (2.2) и (1.4) статьи [ $\left.{ }^{8}\right]$, получаем более простые доказательства достаточности условий теоремы 2 статьи [6] и теоремы 4 статьи [7]. В частности, отсюда получаем обобщения последних теорем и для $\alpha, \beta>-1$ при $\gamma, \delta \leqslant \alpha+1, \beta+1$, так как необ-

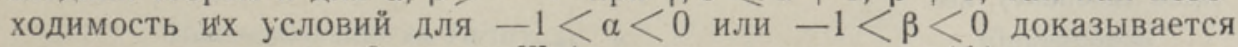
так же, как в лемме 2 статьи [6] (если вместо услови'я (А) использовать условие $\left.\left(\mathrm{M}^{3}\right)\right)$.

\section{ЛИТЕРАТУ РА}

1. L. S. Bos anquet, H. C. Chow, Some remarks on convergence and summability factors. J. London Math. Soc., 1957, 32, 73-82.

2. H. C. Chow, Note on convergence and summability factors. J. London Math. Soc., $1954,29,459-476$.

3. A. Pe yerimh of f, Ober ein Lemma von Herrn H. C. Chow. J. London Math. Soc., $1957,32,33-36$.

4. С. Барон, Новые доказательства основных теорем о множителях суммируемости. Изв. АН ЭССР, сер. физ.-мат. и техн, наук, т. IX, № 1, 1960.

5. С Б арон, Множители суммируемости и абсолютной суммируемости для двой. ных рядов, абсолютно суммируемых методом Чезаро, Уч. зап. Тартуск. ун-та, $1961,102,118-134$. 
6. С. Ба рон, Множители абсолютной суммируемости для Чезаро-суммируемых и Чезаро-ограниченных двойных рядов. Уч. зап. Тартуск. ун-та, 1961, 102, $135-155$

7. С. Барон, Т. Т а м м ӓ,, О множителях суммируемости для метода Чезаро отрицательного порядка. Изв. АН ЭССР, сер. физ.-мат. и техн. наук, т. ХI, № 1, 1962.

8. И. Е. Ж а к, М. Ф. Т и м ан, О суммировании двойных рядов. Матем. сб., 1954. $35(77), 21-56$.

9. Г. К а г ро, С. Ба рон, Множители суммируемости для Чезаро-суммируемых и Чезаро-ограниченных двойных рядов. Уч. зап. Тартуск. ун-та, 1959, 73, 3-49.

10. Г. Х а р д и, Расходящиеся ряды, М., 1951.

11. G. D. Dikshit, On the absolute summability factors of infinite series. Proc. Nat. Inst. Sci. India, 1959, A25, 191-200.

12-14. T. P a ti, Z. U. A h m d, On the absolute summability factors of infinite series (I-III). Tohoku Math. J., 2, 1960, 12, 222 - 232; Indian J. Math., 1960, 2, 29-39; Indian J. Math., 1960, 2, 73-87.

15. Z. U. A h m a d, On the absolute Cesàro summability factors of infinite series. Math. Z., 1961, 76, 295-310.

Тартуский государственный университет Институт кибернетики

Академии наук Эстонской ССР

Поступила в редакщию

3. VI 1961

\title{
H. C. CHOW' KAHEST TEOREEMIST JA NENDE OLDISTUSTEST KAHEKORDSETELE RIDADELE
}

\author{
S. Baron, \\ füüsikalis-matemaatiliste teaduste kandidaat \\ E. Pallum, M. Peterson \\ Resümee
}

Olgu $\left\{\varphi_{n}\right\}$ mittekasvav positiivsete arvude jada, $\alpha \geqslant 0, \beta>-1$.

Rida

$$
\Sigma u_{n}
$$

nimetame $\mid C_{\varphi}^{\alpha}$ |-summeeruvaks, kui $\sum_{-} \varphi_{n}\left|u_{n}^{\prime}\right|<\infty$. kus

$$
u_{n}^{\prime}=\sum_{k=0}^{n} \frac{k}{n} \frac{A_{n-k}^{\alpha-1}}{A_{n}^{\alpha}} u_{k}
$$

Jada $\left\{U_{n}\right\}$ nimetame $C_{\varphi}^{\alpha}$-tôkestatuks $\left(C_{\varphi}^{\alpha}\right.$-summeeruvaks), kui jada $\left\{\varphi_{n} U_{n}^{\prime}\right\}$ on tōkes. tatud (koonduv), kus

$$
U_{n}^{\prime}=\sum_{k=0}^{n} \frac{A_{n-k}^{\alpha-1}}{A_{n}^{\alpha}} U_{k} .
$$

Käesolevas artiklis antakse tarvilikud ja piisavad tingimused selleks, et

1) rea (1) $\left|C_{\varphi}^{\alpha}\right|$-summeeruvusest järelduks rea

$$
\stackrel{\Sigma}{\Sigma} u_{n}
$$

$C^{\beta}$ - või $\left|C^{\beta}\right|$-summeeruvus (teoreem 1);

2) jada $\left\{n u_{n}\right\} \quad C_{\varphi}^{\alpha}$-tōkestatusest või $C_{\varphi}^{\alpha}$-summeeruvusest järelduks rea $\mathrm{i}^{\beta} \mid$ - summeeruvus (teoreem 2).

Teoreemid 1 ja 2 üldistatakse ka kahekordsetele ridadele (teoreemid 3 ja 4).

Tartu Riiklik Olikool

Eesti NSV Teaduste Akadeemia

Küberneetika Instituut
Saabus toimetusse

3. VI 1961 


\section{OBER ZWEI SÄTZE VON H. C. CHOW \\ UND IHRE VERALLGEMEINERUNGEN AUF DOPPELREIHEN}

\section{S. Baron, E. Pallum, M. Peterson}

\section{Zusammenfassung}

Es sei $\left\{\varphi_{n}\right\}$ eine nichtwachsende positive Zahlenfolge, und $\alpha \geqslant 0, \beta>-1$.

Wir nennen eine Reihe

$$
\sum u_{n}
$$

$\left|C_{\varphi}^{\alpha}\right|$-summierbar, wenn $\Sigma_{\varphi_{n}}\left|u_{n}^{\prime}\right|<\infty$, wo

$$
u_{n}^{\prime}=\sum_{k=0}^{n} \frac{k}{n} \frac{A_{n-k}^{\alpha-1}}{A_{n}^{\alpha}} u_{k} .
$$

Wir nennen eine Folge $\left\{U_{n}\right\} C_{\varphi}^{\alpha}$-beschränkt ( $C_{\varphi}^{\alpha}$-summierbar), wenn die Folge $\left\{\Phi_{n} U_{n}^{\prime}\right\}$ beschränkt (konvergent) ist, wo

$$
U_{n}^{\prime}=\sum_{k=0}^{n} \frac{A_{n-k}^{\alpha-1}}{A_{n}^{\alpha}} U_{k} .
$$

Es ist das Ziel dieser Arbeit, notwendige und hinreichende Bedingungen anzugeben, unter welchen

1) für alle $\left|C_{\varphi}^{\alpha}\right|$-summierbaren Reihen (1) die Reihe

$$
\Sigma \varepsilon_{n} u_{n}
$$

$C^{\uparrow}$ - oder $\left|C^{\beta}\right|$-summierbar wäre (Theorem 1);

2) für alle $C_{\varphi}^{\alpha}$-beschränkten oder $\ddot{C}_{\varphi}^{\alpha}$ stimmierbaren Folgen $\left\{n u_{n}\right\}$ die Reihe (2) $\left|C^{\beta}\right|$-summierbar wäre (Theorem 2).

Diese Theoreme werden auch für Doppelreihen verallgemeinert (Theoreme 3 und 4).

\section{Staatsuniversität zu Tartu}

$$
\text { Institut für Kybernetik }
$$

der Akademie der Wissenschaften der Estnischen SSR

Eingegangen am 3. Juni 1961 